\title{
OANI PHTTOOPTICS
}

Global chlorophyll a concentrations of phytoplankton functional types with detailed uncertainty assessment using multi-sensor

ocean color and sea surface temperature products

- EOF-PFT algorithm v2

$\underline{\text { H Xi }}^{1}$, SN Losa ${ }^{1,2}$, A Mangin ${ }^{3}$, P Garnesson ${ }^{3}$, M Bretagnon ${ }^{3}$, J Demaria ${ }^{3}$, MA Soppa ${ }^{1}$, OHF d'Andon ${ }^{3}$, A Bracher ${ }^{1,4}$

${ }^{1}$ Alfred Wegener Institute, Helmholtz Centre for Polar and Marine Research

2 Shirshov Institute of Oceanology, Russian Academy of Sciences

${ }^{3} \mathrm{ACRI}-\mathrm{ST}$

4 Institute of environmental Physics, University of Bremen 


\section{Motivation}

- Within the frame of a collaborative project OLCI-PFT (2018-2020) https://www.awi.de/en/science/climate-sciences/physical-oceanography/main-research-focus/ocean-optics/projects/olci-pft.html

- Project Goal:

Globally retrieving multiple phytoplankton functional types (PFTs) from multisensor ocean color products and Sentinel-3 OLCl data

- Long-term consistent PFT monitoring on both global and regional scales

- Support more accurate ecosystem modelling 


\section{Objectives of the current study}

I. Improve the previously developed algorithm for retrieving phytoplankton functional types (PFT) (EOF-PFT v1 $\rightarrow$ v2)

II. Provide per-pixel uncertainty for the satellite PFT products

III. Extend EOF-PFT approach to different ocean color sensors to obtain continuous PFT observations from space 


\section{Data sets}

- global database of in situ HPLC pigments for PFT $(N>17,000)$
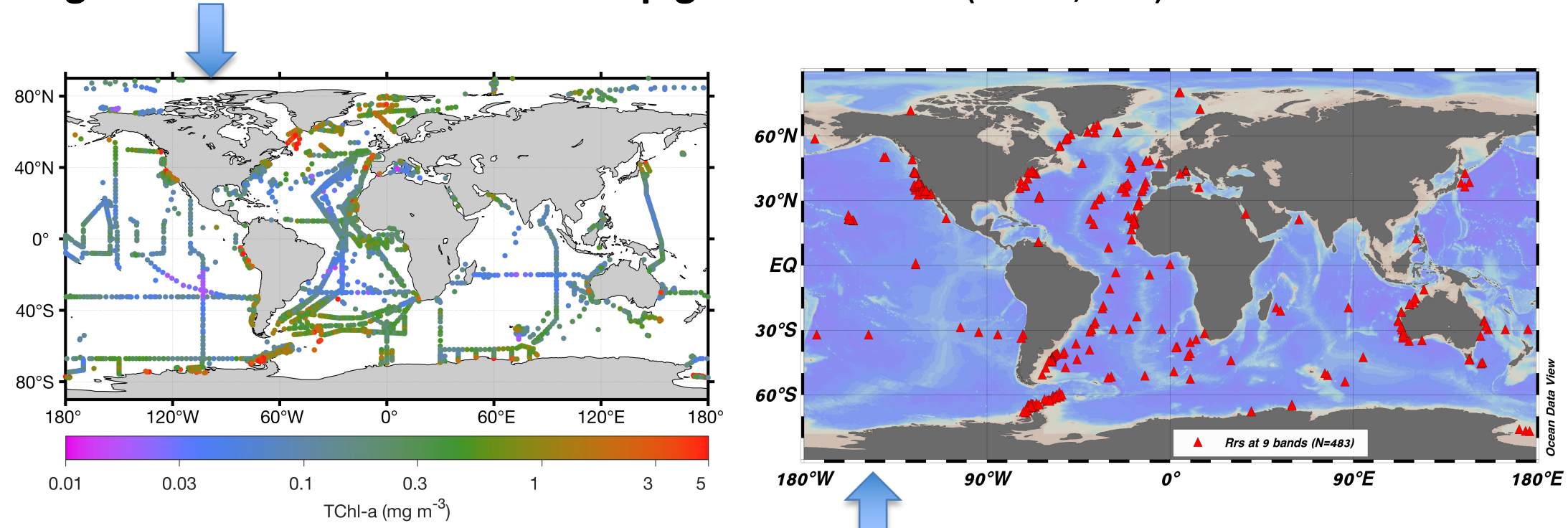

- Matchups between satellite reflectance (Rrs), in situ PFT data, and sea surface temperature (SST)

- Merged OC products SeaWiFS-MODIS-MERIS (2002-2012), MODIS-VIIRS (2012present), and OLCI data (2016-present), CMEMS OSTIA SST products 


\section{Algorithm}

- Re-tuned PFT Algorithm EOF-PFT v2.0

\section{Input data for model establishment:}

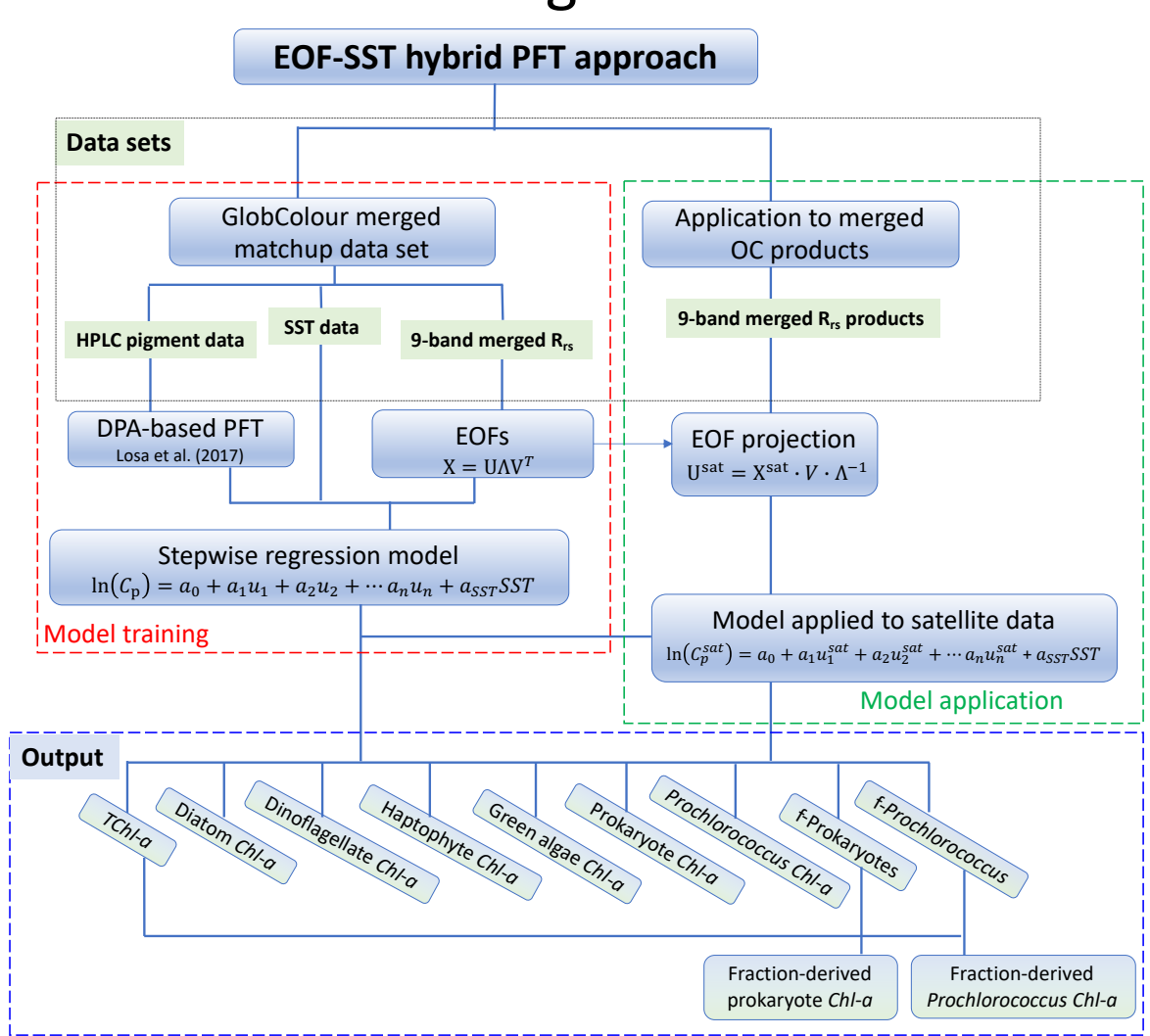

- In situ phytoplankton functional types (PFT) data

- Satellite Remote sensing reflectance (Rrs)

- Sea surface temperature

\section{Method}

- Empirical orthogonal function (EOF) analysis in Rrs

- Regression model for PFT: In situ PFT as a function of EOFs derived from Rrs and SST

\section{Input data for application to satellites}

- Satellite Rrs products (global)

- Satellite SST products (global)

\section{Final Output}

- Total chlorophyll concentration (TChl-a, total biomass)

- 6 different PFTs: diatoms, haptophytes etc.

- 2 fractions of picophytoplankton

5 Flowchart of EOF-PFT v2.0 based on SeaW-MOD-MER merged products 


\section{Uncertainty sources and error propagation}

The final PFT retrieval model:

$y\left(\boldsymbol{a}, \boldsymbol{u}\left(R_{r s}\right), S S T\right)=\ln \left(C_{P}^{s a t}\right)=a_{o}+a_{1} u_{1}^{s a t}+a_{2} u_{2}^{s a t}+\cdots a_{n} u_{n}^{s a t}+a_{S S T} S S T$

Model parameters

EOF scores derived from satellite

Rrs

$\boldsymbol{a}, \boldsymbol{u}$, and SST in Eq. (3) are not correlated with each other, the uncertainty of the PFT is presented as:

$$
\begin{aligned}
& \sigma_{\mathrm{y}}=\sqrt{\sigma_{y(R r s)}^{2}+\sigma_{y(a)}^{2}+\sigma_{y(S S T)}^{2}}=\sqrt{\sum_{i=1}^{N}\left(\frac{\partial y}{\partial R r s_{i}}\right)^{2} \sigma_{R r s_{i}}^{2}+\sum_{i=0}^{n}\left(\frac{\partial y}{\partial a_{i}}\right)^{2} \sigma_{a_{i}}^{2}+\left(\frac{\partial y}{\partial S S T}\right)^{2} \sigma_{S S T}^{2}} \\
& \sigma_{y(a)}^{2}+\sigma_{y(S S T)}^{2}=\sum_{i=0}^{N}\left(\frac{\partial y}{\partial a_{i}}\right)^{2} \sigma_{a_{i}}^{2}+\left(\frac{\partial(y)}{\partial a_{S S t}}\right)^{2} \sigma_{a_{S S t}}^{2}+\left(\frac{\partial(y)}{\partial S S T}\right)^{2} \sigma_{S S T}^{2}
\end{aligned}
$$

$\sigma_{y(R r s)}$ is not as straightforward as the other two uncertainty sources

due to nonlinear transformation from the Rrs spectra.

Monto Carlo simulation is performed. 
Statistical results of EOF-PFT v2.0 versus v1.0

\begin{tabular}{lccccccc}
\hline & $\mathrm{N}$ & $\begin{array}{c}\mathrm{MDPD} \\
(\%)\end{array}$ & $\begin{array}{l}\mathrm{RMSD} \\
\left(\mathrm{mg} \mathrm{m}^{-3}\right)\end{array}$ & $\mathrm{R}^{2}$ & $\begin{array}{l}\text { MDPDcv } \\
(\%)\end{array}$ & $\begin{array}{l}\text { RMSDcv } \\
\left(\mathrm{mg} \mathrm{m}^{-3}\right)\end{array}$ & $\mathrm{R}^{2} \mathrm{cv}$ \\
\hline EOF-SST hybrid model & EOF-PFT V2.0 & & & & & \\
TChl-a & 483 & $\mathbf{3 1 . 5 5}$ & $\mathbf{1 . 0 8}$ & $\mathbf{0 . 8 2}$ & $\mathbf{3 1 . 5 1}$ & $\mathbf{1 . 1 4}$ & $\mathbf{0 . 8 1}$ \\
Diatoms & 345 & $\mathbf{5 6 . 2 5}$ & $\mathbf{0 . 9 2}$ & $\mathbf{0 . 7 7}$ & $\mathbf{5 7 . 7 1}$ & $\mathbf{0 . 9 8}$ & $\mathbf{0 . 7 6}$ \\
Dinoflagellates & 295 & $\mathbf{5 4 . 0 0}$ & $\mathbf{0 . 8 9}$ & $\mathbf{0 . 6 2}$ & $\mathbf{5 4 . 6 8}$ & $\mathbf{0 . 7 1}$ & $\mathbf{0 . 6 0}$ \\
Haptophytes & 454 & $\mathbf{4 2 . 8 8}$ & $\mathbf{0 . 1 6}$ & $\mathbf{0 . 7 1}$ & $\mathbf{4 3 . 6 3}$ & $\mathbf{0 . 1 7}$ & $\mathbf{0 . 6 9}$ \\
Green algae & 323 & $\mathbf{5 1 . 5 7}$ & $\mathbf{0 . 1 0}$ & $\mathbf{0 . 5 3}$ & $\mathbf{5 2 . 9 6}$ & $\mathbf{0 . 1 1}$ & $\mathbf{0 . 5 1}$ \\
Prokaryotes & 438 & $\mathbf{4 3 . 7 9}$ & $\mathbf{0 . 0 9}$ & $\mathbf{0 . 4 2}$ & $\mathbf{4 5 . 4 4}$ & $\mathbf{0 . 0 9}$ & $\mathbf{0 . 3 8}$ \\
f-Prokaryotes & 441 & 47.35 & $0.21 *$ & 0.70 & 47.72 & $0.21 *$ & 0.68 \\
Fraction-derived prokaryotes & 434 & $\mathbf{4 2 . 1 3}$ & $\mathbf{0 . 0 9}$ & $\mathbf{0 . 4 6}$ & & & \\
Prochlorococcus & 204 & 42.60 & 0.02 & 0.24 & 44.97 & 0.02 & 0.17 \\
f-Prochlorococcus & 210 & 41.52 & $0.09 *$ & 0.62 & 43.99 & $0.09 *$ & 0.57 \\
Fraction-derived Prochlorococcus & 198 & 39.90 & 0.02 & 0.24 & & & \\
Original EOF-based algorithm & EOF-PFT v1.0 & & & & & \\
TChl-a & 394 & 37.41 & 1.24 & 0.76 & 37.08 & 1.27 & 0.75 \\
Diatoms & 306 & 73.70 & 1.21 & 0.65 & 74.74 & 1.29 & 0.63 \\
Dinoflagellates & 272 & 55.32 & 0.93 & 0.62 & 57.29 & 0.72 & 0.59 \\
Haptophytes & 387 & 47.16 & 0.22 & 0.64 & 48.62 & 0.24 & 0.61 \\
Green algae & 262 & 55.81 & 0.11 & 0.51 & 56.26 & 0.11 & 0.48 \\
Prokaryotes & 367 & 53.70 & 0.13 & 0.15 & 55.08 & 0.13 & 0.11 \\
Prochlorococcus & 142 & 39.65 & 0.02 & 0.24 & 42.68 & 0.02 & 0.18 \\
\hline
\end{tabular}
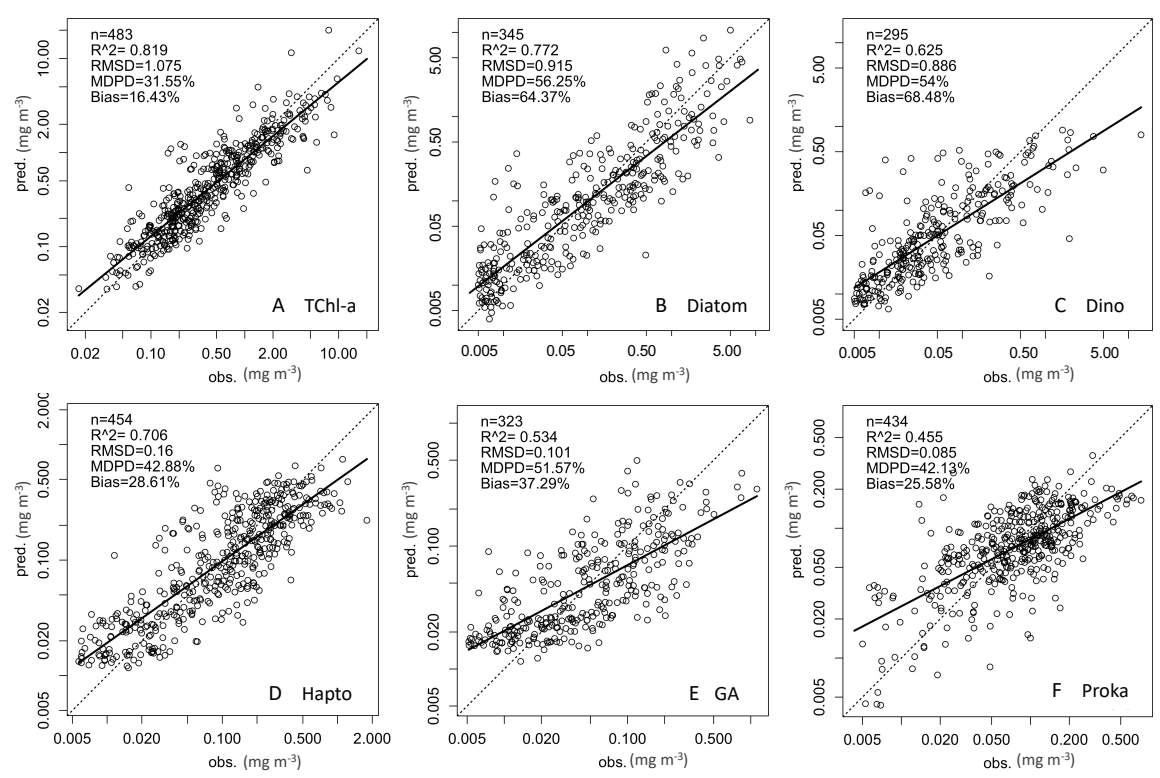

Examples of regressions between observed ( $\mathrm{x}$-axis, obs.) and predicted (y-axis, pred.) PFT quantities using EOF-PFT v2.0 


\section{PFT products from EOF-PFT v2.0}

Satellite-derived estimates of annual (2011) mean surface TChl-a and Chl-a concentrations of six PFTs
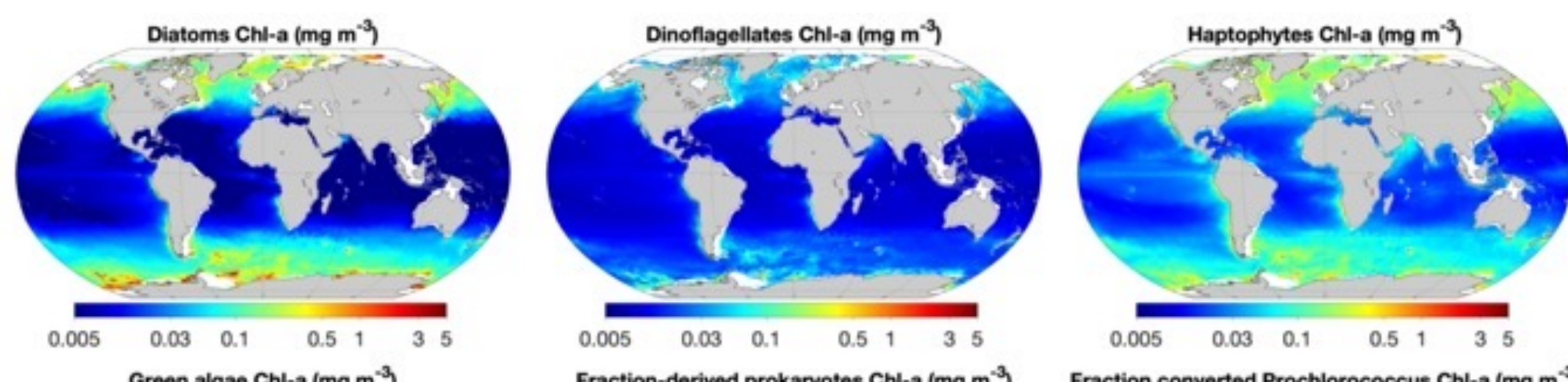

Fraction-derived prokaryotes $\mathrm{Chl}-\mathrm{a}\left(\mathrm{mg} \mathrm{m}^{-3}\right)$
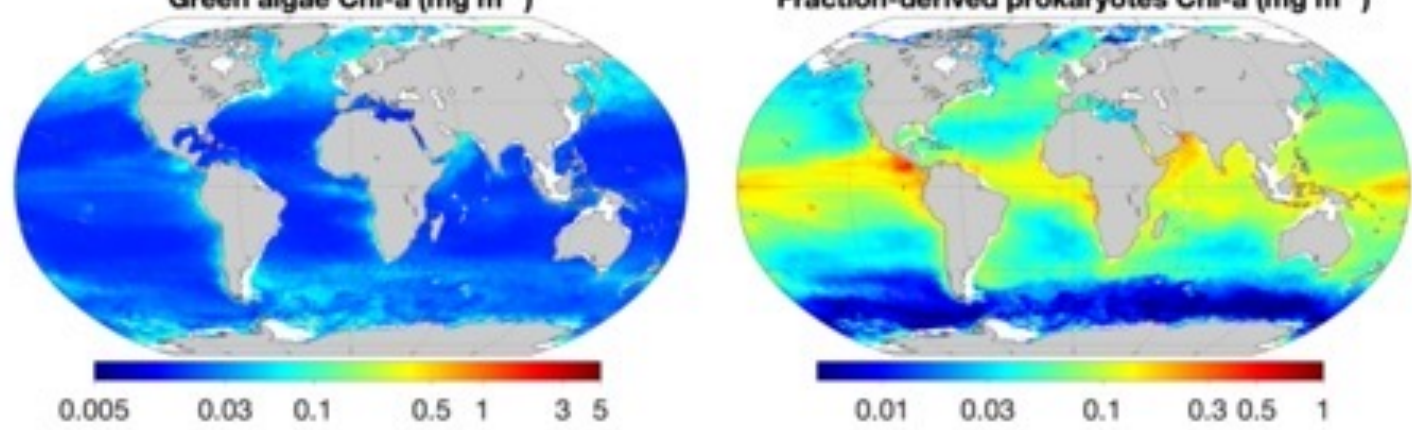

Fraction converted Prochlorococcus Chl-a ( $\mathrm{mg} \mathrm{m}^{-3}$ )

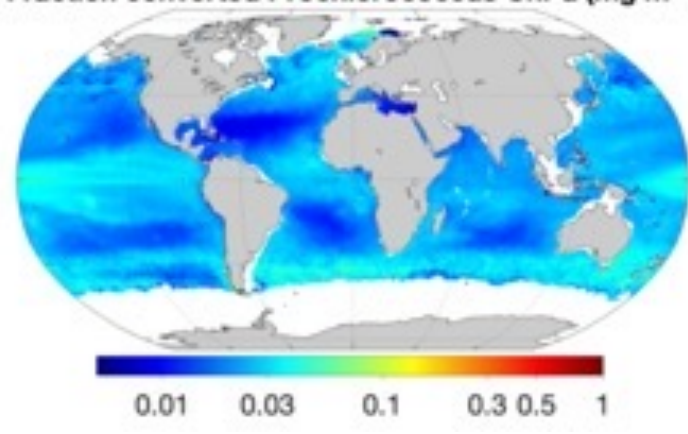




\section{PFT uncertainty from EOF-PFT v2.0}

Per-pixel uncertainty (in natural logarithmic scale) of the annual mean of 2011 for the satellite-derived PFT quantities from EOF-PFT v2.0
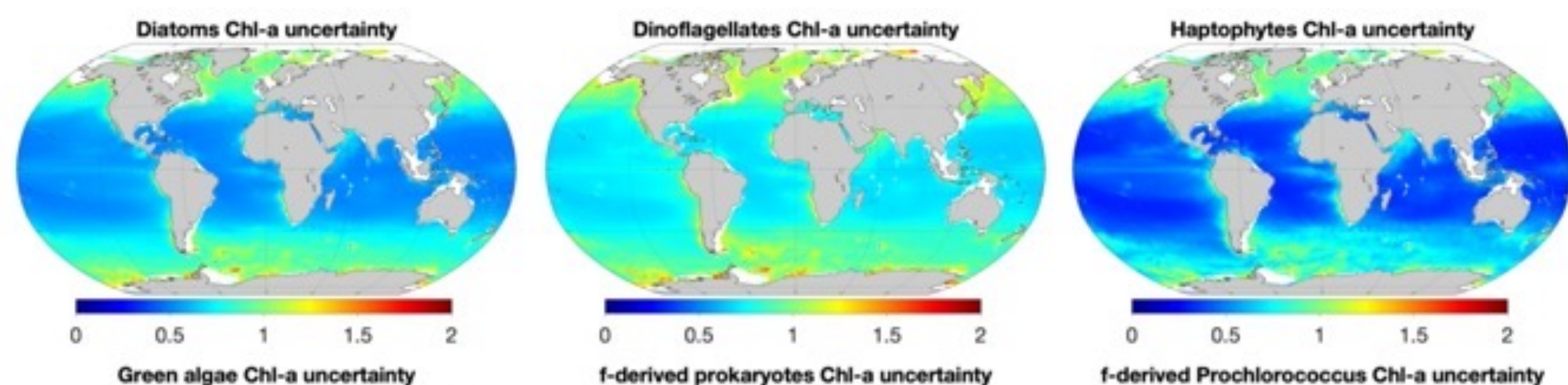

Green algae Chl-a uncertainty
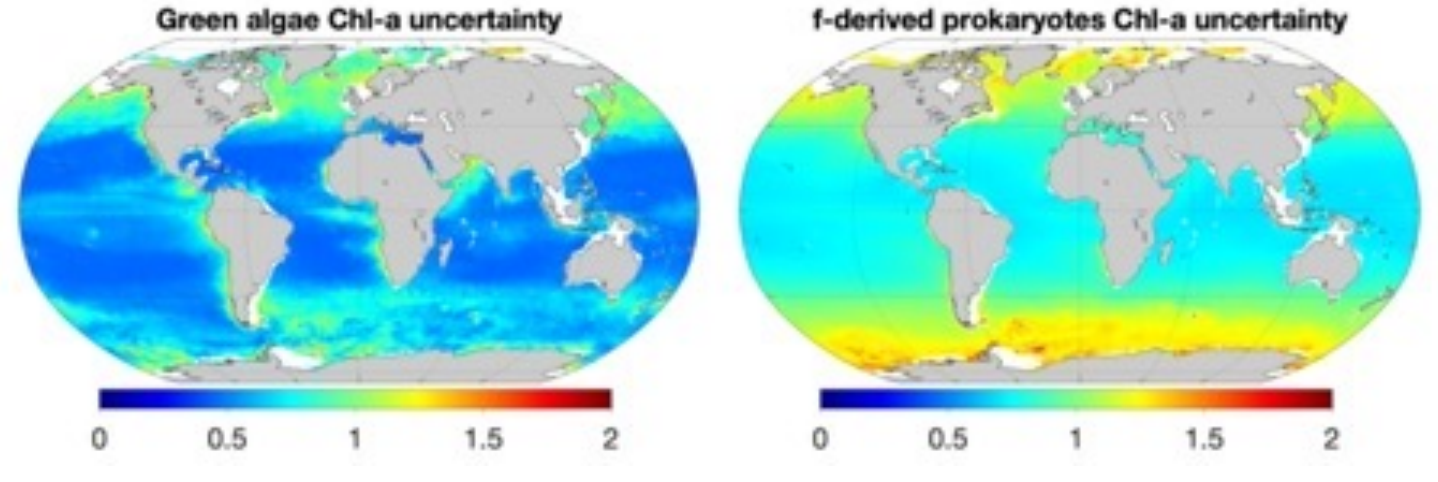

f-derived Prochlorococcus Chl-a uncertainty
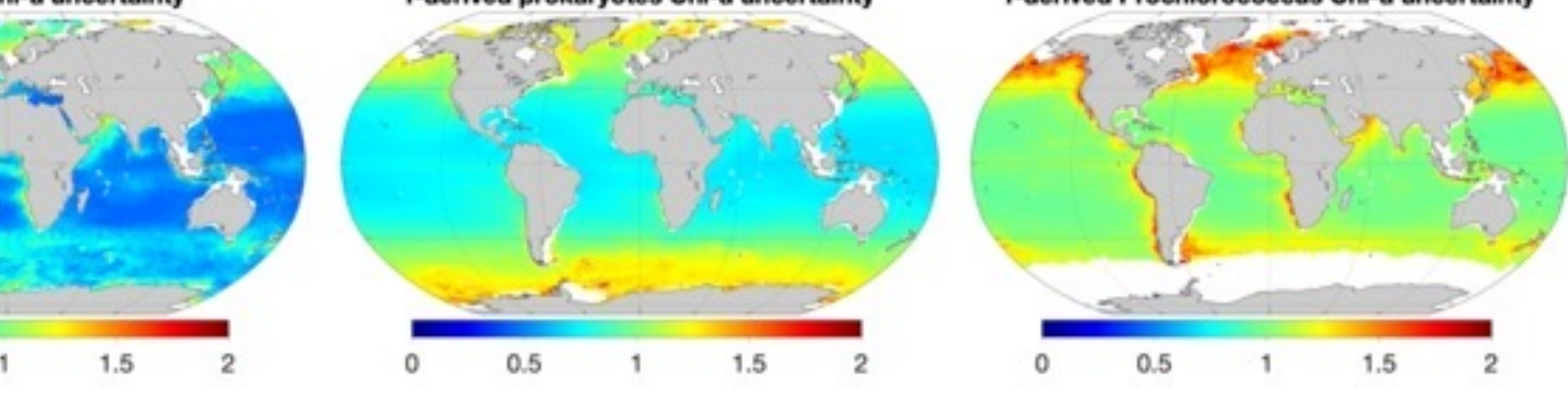


\section{Uncertainty comparison}
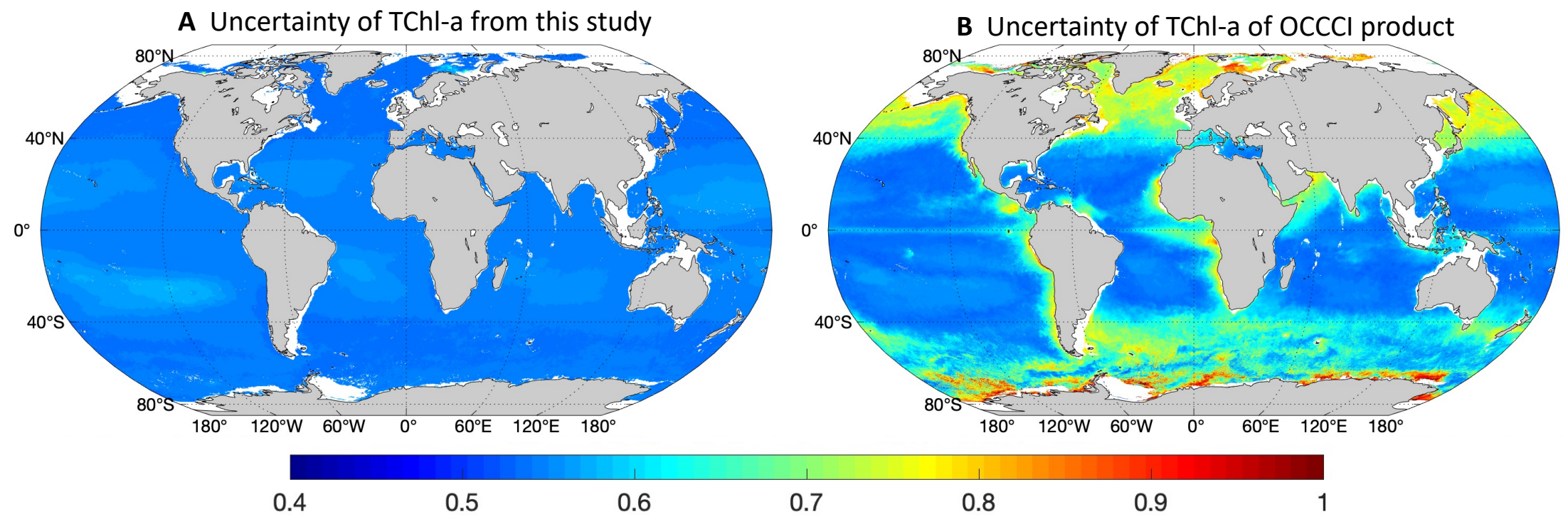

EOF-PFT v2.0: 52\% - 58\%

OC-CCl: $43 \%$ - 118\%

- TChl-a uncertainty derived from EOF-PFT v2.0 is in general lower than the OC-CCl standard product

- First time the per-pixel PFT uncertainty description on a global scale 
Extended versions v2.1 and v2.2

- EOF-PFT v2.1 for MOD-VIR merged products

- EOF-PFT v2.2 for OLCl products 


\section{EOF-PFT v2.1}

\section{Application to MOD/VIIRS 201801}

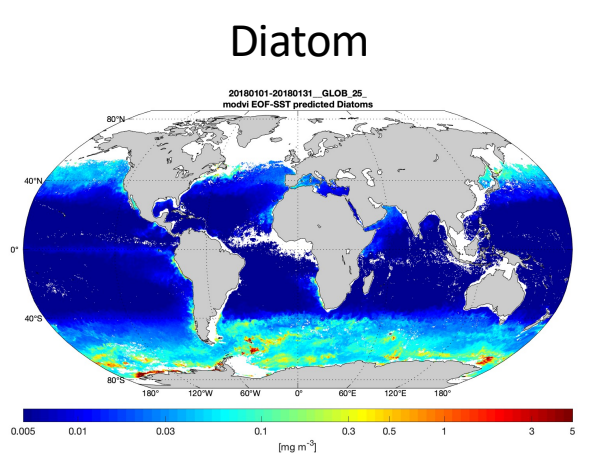

Green algae

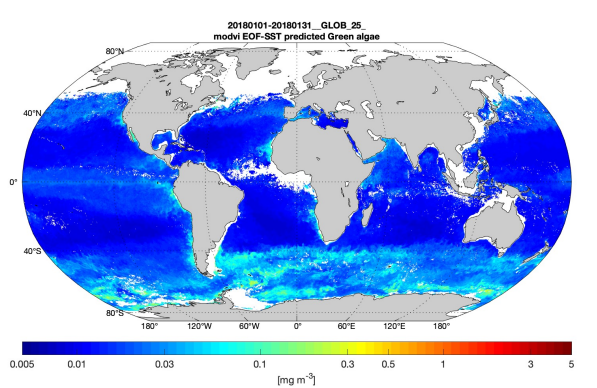

Dinoflagellates

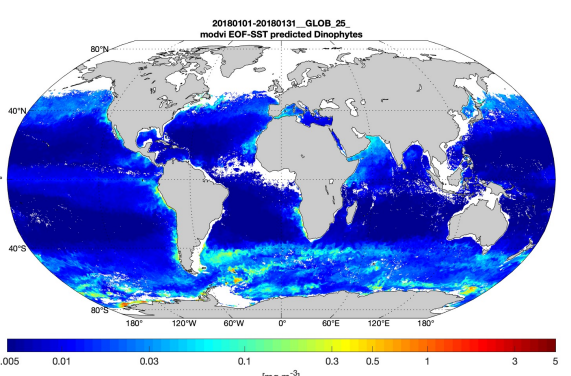

Prokaryotes

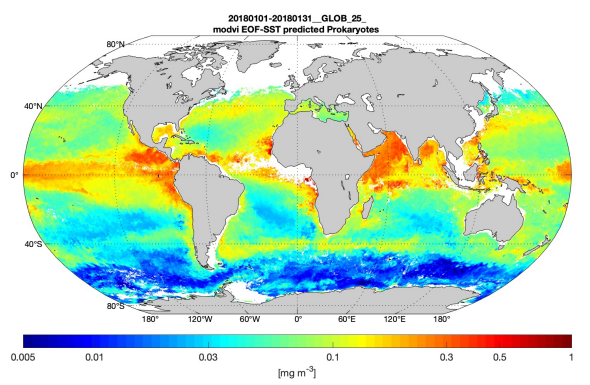

Haptophytes

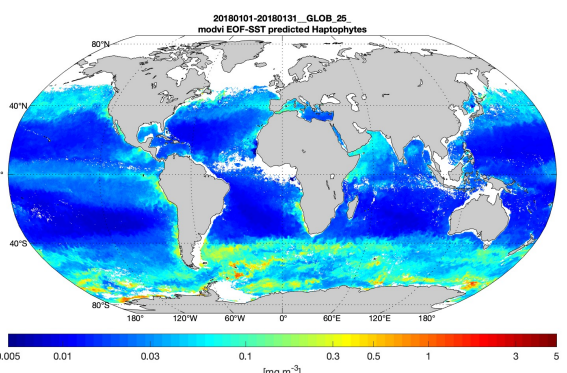

Prochlorococcus

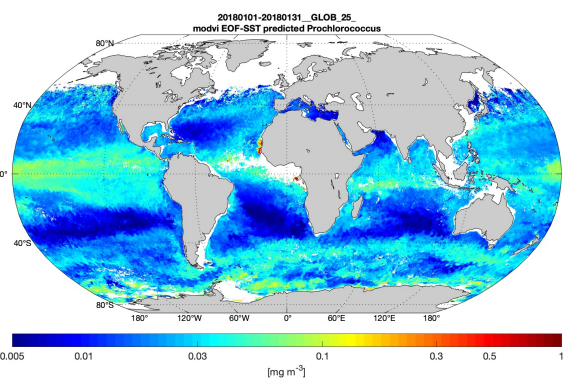




\section{Application to OLCI 201801}

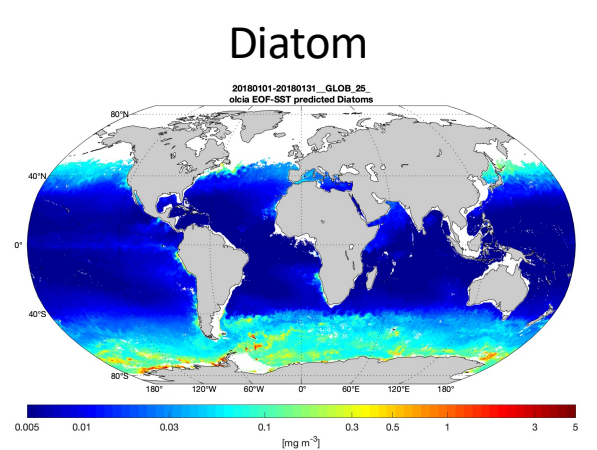

Green algae

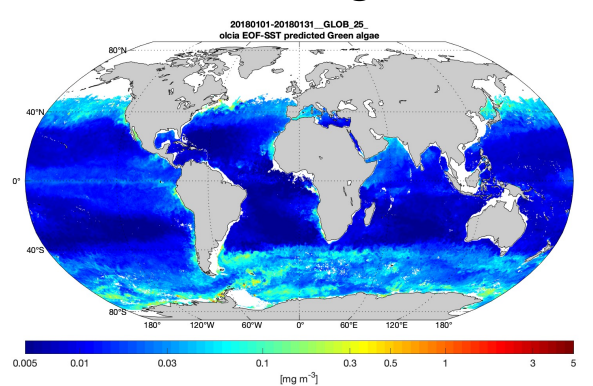

Dinoflagellates

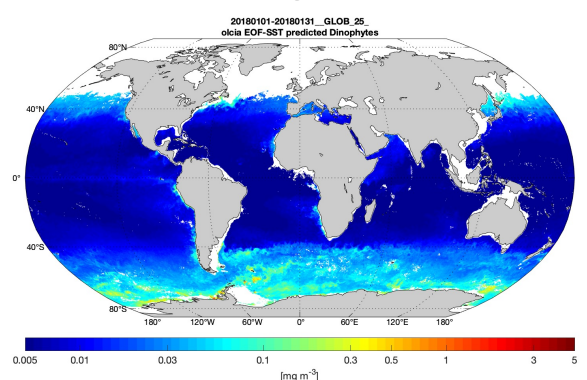

Prokaryotes

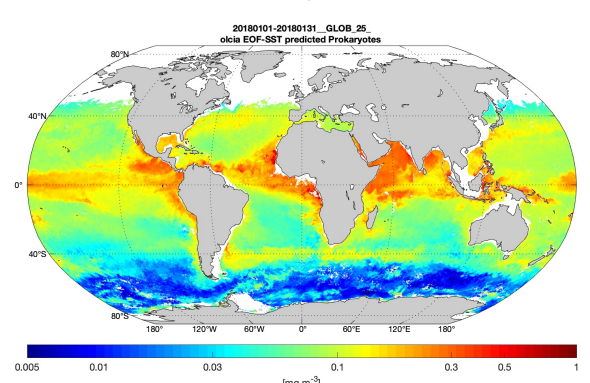

Haptophytes

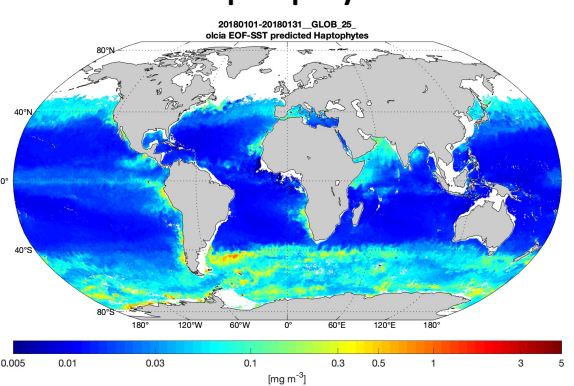

Prochlorococcus

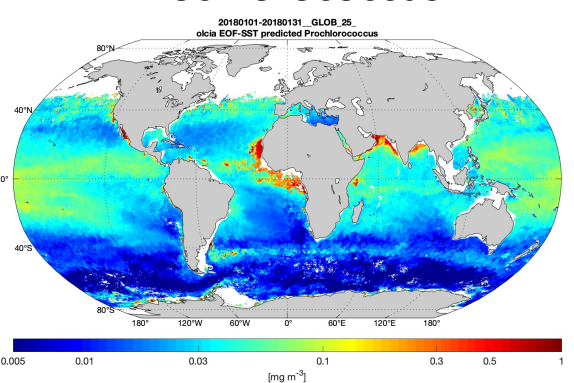




\section{Product consistency}

\section{EOF-PFT v2.0 versus 2.1}
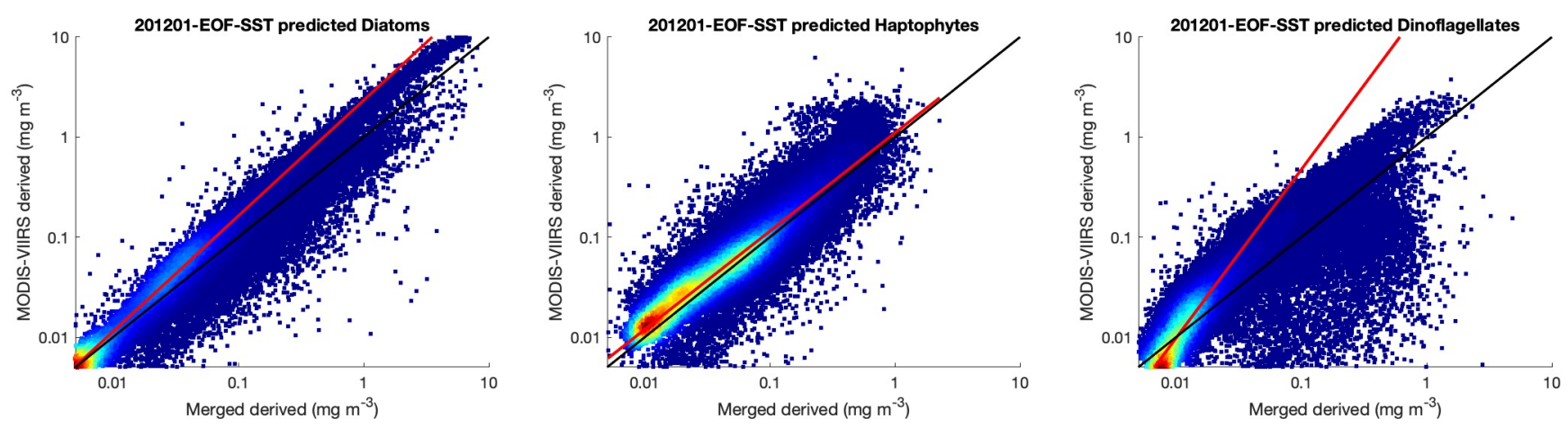

Diatoms
Dinoflagellates

Haptophytes

Green algae

Proka_converted

Prochlo-converted

\begin{tabular}{|c|c|c|}
\hline Slope & Intercept & $\mathrm{R}^{2}$ \\
\hline 1.16 & 0.37 & 0.96 \\
\hline 1.68 & 1.36 & 0.82 \\
\hline 0.98 & 0.04 & 0.93 \\
\hline 1.41 & 0.72 & 0.78 \\
\hline 0.98 & 0.08 & 0.86 \\
\hline 1.08 & 0.35 & 0.41 \\
\hline
\end{tabular}
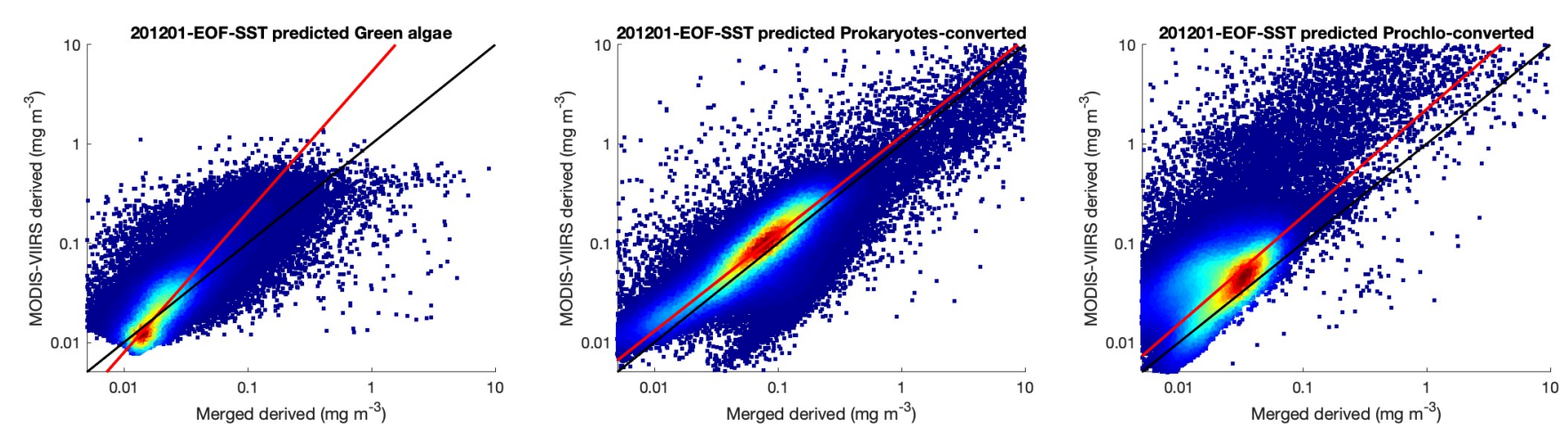

Statistics of type II Regression with uncertainty accounted between MOD/VIR and SeaW/MOD/MER monthly PFTs for Jan 2012. 


\section{List of EOF-PFT algorithm versions}

\begin{tabular}{|c|c|c|c|c|c|c|}
\hline & \begin{tabular}{|l} 
EOF-PFT \\
version
\end{tabular} & $\begin{array}{l}\text { Date of } \\
\text { development }\end{array}$ & Applicable sensor(s) & Input data & \begin{tabular}{|l} 
Product \\
time frame
\end{tabular} & Remarks \\
\hline \multirow[t]{3}{*}{$\begin{array}{l}\text { EOF-based } \\
\text { algorithm }\end{array}$} & v1.0 & Dec 2019 & SeaWiFS/MODIS/MERIS merged product & 9-band merged $R_{r s}$ & $2002-2012$ & $\begin{array}{l}\text { In situ data from } \\
\text { Losa et al. (2017) }\end{array}$ \\
\hline & v1.1 & Jan 2019 & MODIS/VIIRS merged product & 9-band merged $\mathrm{R}_{\mathrm{rs}}$ & 2012-present & $\begin{array}{l}\text { Losa et al. (2017) } \\
\text { and own cruises }\end{array}$ \\
\hline & v1.2 & Jan 2019 & Sentinel 3 OLCI-A product & 11-band OLCI Rrs & 2016-present & $\begin{array}{l}\text { In situ data from } \\
\text { own cruises }\end{array}$ \\
\hline \multirow{3}{*}{$\begin{array}{l}\text { EOF-SST } \\
\text { hybrid } \\
\text { algorithm }\end{array}$} & v2.0 & Mar 2020 & SeaWiFS/MODIS/MERIS merged product & $\begin{array}{l}\text { 9-band merged } \mathrm{R}_{\mathrm{rs}} \\
\text { SST }\end{array}$ & $2002-2012$ & $\begin{array}{l}\text { Updated pigment } \\
\text { data }\end{array}$ \\
\hline & v2.1 & May 2020 & MODIS/VIIRS merged product & $\begin{array}{l}\text { 9-band merged } \mathrm{R}_{\mathrm{rs}} \\
\text { SST }\end{array}$ & 2012-present & $\begin{array}{l}\text { Updated pigment } \\
\text { data }\end{array}$ \\
\hline & v2.2 & May 2020 & Sentinel 3 OLCI-A product & $\begin{array}{l}\text { 11-band OLCI Rrs, } \\
\text { SST }\end{array}$ & 2016-present & $\begin{array}{l}\text { Updated pigment } \\
\text { data }\end{array}$ \\
\hline $\begin{array}{l}\text { SST-separated } \\
\text { hybrid } \\
\text { algorithm }\end{array}$ & v3.0 & Jul 2020 & SeaWiFS/MODIS/MERIS merged product & $\begin{array}{l}\text { 9-band merged } R_{r S} \\
\text { SST }\end{array}$ & $2002-2012$ & $\begin{array}{l}\text { Updated pigment } \\
\text { data }\end{array}$ \\
\hline
\end{tabular}




\section{Algorithm and Products}

Available online

- Algorithm implemented into Coastal-TEP : https://www.coastal-tep.eu/geobrowser/

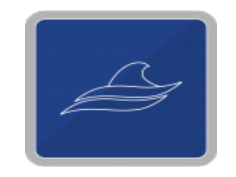

- Data products available in CMEMS Under "Ocean Products" at https://marine.copernicus.eu/

Updated version available online in May 2021 


\section{Summary and Outlook}

- Improved satellite PFT products are provided using updated algorithms EOF-PFT v2

- Per-pixel uncertainty of the satellite PFT products is assessed in detail by accounting for errors from input data and model parameters

- Extendable to other ocean color satellite products

Outlook:

- Continuous time series of global PFT - long term monitoring

- PFT shifting and trend under the changing climate

For more detailed information please check on the publication by Xi et al. 2021: https://doi.org/10.1029/2020JC017127 


\section{Acknowledgements}

- OLCI-PFT Project (ACRI-AWI Offer \#209-180104)

- Deutsche Forschungsgemeinschaft (DFG, German Research Foundation)-Project number 268020496-TRR 172, within the Transregional Collaborative Research Center ArctiC Amplication: Climate Relevant Atmospheric and SurfaCe Processes, and Feedback Mechanisms (AC) ${ }^{3}$ (Project C03)

- Federal Agency for Scientific Organizations (FASO) Russia (theme 0128-2021-0014)

- Marc Taylor for original EOF script, Sonja Wiegmann and all previous and current Phytooptics team members who participated in the past cruises for data collection and analysis

- All the scientists and crew who were involved in the global HPLC pigment data collection and analyses for making their data publicly available

- NASA, ESA, EUMETSAT for the SeaWiFS, MODIS, MERIS and OLCI data

- GlobColour program for the L3 merged products

- CMEMS for the sea surface temperature products

- Ocean-Colour Climate Change Initiative (OC-CCI) program for global chlorophyll-a data products 